\title{
Is my PET in my genes?
}

\author{
Mallory Downie $^{1} \cdot$ Rukshana Shroff $^{2,3} \cdot$ Detlef Bockenhauer $^{1,2}$ (])
}

Received: 21 December 2021 / Revised: 28 December 2021 / Accepted: 28 December 2021 / Published online: 25 January 2022

(c) The Author(s), under exclusive licence to International Pediatric Nephrology Association 2022

\section{The quest for precision}

Precision medicine holds the promise of individualized care. Thus, while traditional evidence-based medicine, with its reliance on large randomized controlled trials, can only provide prognostic and treatment information for a defined group of patients, precision medicine aims to go beyond that, by providing accurate information on treatment response and prognosis for each individual patient. Thereby, precision medicine tries to address the problem of the large spectrum of severity within a given clinical group, by identifying the genetic and environmental factors that contribute to the phenotypic diversity. Imagine that you could with reasonable accuracy predict beforehand whether a patient with, for instance, $\operatorname{Ig}$ A nephropathy will eventually have spontaneous resolution or progressive chronic kidney disease, whether this will respond to immunosuppression and, if so, which drug will work best with the least side effects! Or, as we will discuss here, whether a patient with kidney failure is likely to do well on peritoneal dialysis (PD) or not and with what kind of PD prescription.

\section{The problem with precision}

The problem, of course, is that despite all the talk, we are still a long way from such precision, although in some areas of medicine, most prominently oncology, enormous strides have been made [1]. Most progress so far has come from genetics, perhaps because of the stability of the genetic code

Detlef Bockenhauer

d.bockenhauer@ucl.ac.uk

1 Department of Renal Medicine, University College London, London, UK

2 Renal Unit, Great Ormond Street Hospital for Children NHS Foundation Trust, Great Ormond Street, London WCN1 3JH, UK

3 Institute for Child Health, University College London, London, UK over a lifetime: whereas our environment constantly changes, our genomes stay essentially the same. And whereas genetic studies initially concentrated on the rare variants with large effects that cause Mendelian disorders, increasingly the focus is now on common variants with smaller effects that contribute to the phenotypic diversity of a given disorder. Typically, this is done by genome-wide association studies (GWAS), in which a large number (nowadays usually more than a million) of common variants are each tested for their association with the trait under investigation [2]. Initially, GWAS concerned primarily complex diseases (those not inherited in Mendelian fashion): examples from the kidney field include membranous and IgA nephropathy, as well as steroid-sensitive nephrotic syndrome (SSNS; reviewed in [3]). Increasingly though, GWAS are also used to investigate complex traits, such as estimated glomerular filtration rate or urinary albumin-to-creatinine ratio [3]. Here, we will discuss 2 recently published genetic investigations into peritoneal membrane function in PD patients.

\section{Pores and the peritoneum}

PD is the most widely used kidney replacement therapy in children worldwide [4]. The individual's peritoneal membrane is used as a "semi-permeable membrane" to transport water and solutes from the blood into the peritoneal space, driving diffusion, convection, and ultrafiltration (UF) by manipulating the osmotic concentration of the dialysis fluid. However, unlike synthetic dialysis membranes used for hemodialysis that have carefully engineered pore size and flux, and where the user can choose between a wide selection of different membranes, the physician must accept and work with an individual's peritoneal membrane characteristics when performing PD.

Peritoneal transport characteristics fit with a three-pore model proposed by Rippe et al., i.e., transport via ultrasmall, small, and large pores [5]. The ultrasmall pores correspond to the water-selective Aquaporin-1 channels (AQP1; radius $2-4 \AA$ ), while the molecular identities of the small 


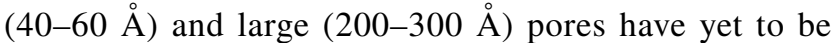
revealed. AQP1 channels make up only $1-2 \%$ of all pores but account for $40-50 \%$ of water transport [6]. The small pores are abundant, making up $90 \%$ of all the peritoneal pores, and allow for 50-60\% of water transport, while water transfer via the large pores is negligible [7]. Of note, the water-selective AQP1 channels mediate water transport only, leading to the phenomenon of sodium sieving, whereas the small pores remove both water and small hydrophilic solutes, such as sodium, by diffusion and convection. Sodium sieving is a consequence of dissociation between the amount of water and sodium transported over the peritoneal membrane resulting from AQP1-mediated water transport [8]. Controlling extracellular volume and plasma sodium concentration are crucial objectives of dialysis therapy, as inadequate sodium and fluid removal by dialysis may result in extracellular volume overload, hypertension, and increased cardiovascular morbidity and mortality.

\section{Predicting pore performance}

Currently, when a patient is started on PD there is no way for the physician to know how that patient's peritoneal membrane will respond in terms of UF and solute clearance. It is only through a trial-and-error process of adapting the PD prescription or by performing serial Peritoneal Equilibration Tests (PET) that their peritoneal transport function can be understood. Thus, for example, some patients fail to achieve adequate UF and are consequently prescribed fluids with high glucose concentration and/or extended dialysis time that can damage the peritoneum, limiting the efficacy and safety of PD. A commonly used measure for peritoneal membrane performance is the peritoneal solute transfer rate (PSTR), which is determined in a PET test and defined as the ratio of creatinine concentration in dialysate and plasma after a 4-h dwell. There is a high interindividual variability in PSTR and this influences the PD prescription. PSTR is also a marker of predicted outcome, as a higher PSTR is associated with higher risk of death and/or hospitalization [9]. Yet, demographic and clinical factors explain only a small proportion of this, raising the possibility that genetic factors may be involved.

And this is exactly what a recent study by Mehrotra et al. investigated [10]. The authors performed a GWAS in a cohort of over 2000 European PD patients to search for associations between any of $>2.3$ million genetic variants and PSTR. When so many statistical associations are explored there needs to be a stringent correction for multiple testing. Since not all variants are truly independent (some are in "linkage disequilibrium," i.e., inherited together), the generally agreed corrected $p$-value for genome-wide statistical significance is $5 \times 10^{-8}$ [11]. Interestingly, despite the strong suggestion of a genetic contribution to the variability in PSTR (the authors calculate that $19 \%$ of the variability is inherited), none of the $>2.3$ million variants assessed reached statistical significance. This suggests that unlike in SSNS or IgA nephropathy, where a few genetic loci (most prominently in the HLA complex) contribute most of the heritability, for PSTR, a larger number of loci with only small effect are responsible. Indeed, the authors identified genetic variants at four loci that showed suggestive $\left(p<1 \times 10^{-5}\right)$ associations with PSTR. Interestingly, two of these genetic loci include genes (KDM2B and $P C H D 9)$ that may influence biological pathways relevant for peritoneal fibrosis and follow-up studies may provide further insights into our understanding of the underlying mechanisms and how to translate that into preventing peritoneal fibrosis [12, 13].

Another study, published almost at the same time, used a different approach: building on the knowledge that AQP1 constitutes the ultrasmall pores important for water transport in PD, Morelle et al. set out to specifically assess variants in $A Q P 1$ and their association with UF [14]. The authors found that variation at rs2075574 (c.781C- $>$ T) in the promoter region of $A Q P 1$ was associated with variability in $\mathrm{UF}$, and that individuals with the risk genotype at this locus had a higher risk of death or PD failure. Expression of AQP1 in the peritoneum of patients with the risk genotype was shown to be lower, in the absence of fibrosis, vascular proliferation, or inflammation. On first read, these findings raise the exciting possibility of genotyping an individual at one genetic variant to predict their UF capabilities. However, as these results are obtained at a population level, conclusions about variation at this marker can only be inferred to populations of specific genotypes, rather than individuals. This becomes immediately apparent when comparing the net UF between patients homozygous for the major or minor allele of rs2075574 (Fig. 1B in [14]): while the median between the 2 groups is significantly different, the range in both groups is substantial and it would be impossible to predict an individual's UF, just based on their rs2075574 genotype.

\section{Precision for PD prescriptions?}

One question that follows from this study is whether adjustment of dialysis prescription could accommodate for the risk genotype. Though the authors do not address this specifically, they do examine the effect of variation at rs2075574 on the type of dialysis fluid used. Interestingly, water flow across the peritoneum generated by icodextrin has been suggested to work independently of aquaporins [15]. Following from this, the authors tested the effect of variation at rs 2075574 on net UF on a subgroup of patients with data on the use of both $3.86 \%$ glucose fluid and icodextrin and found 
that the risk genotype was associated with lower UF when using 3.86\% PD fluid but not when using icodextrin. These results suggest that use of colloid osmotic agents may mitigate the risk associated with the rs 2075574 variant. This is a practical and clinically useful conclusion, though in practice, if PD is difficult or unsuccessful with glucose-based dialysis fluid alone, most nephrologists would add in an icodextrin exchange anyway, irrespective of that individual's genotype.

The association of $A Q P 1$ genotype with UF and patient outcome supports the role of fluid overload in driving poor outcomes for patients on dialysis. There is potential to use this information toward individualized care, particularly if researchers can prospectively group patients by genotype when designing clinical trials in PD. Further to this, combining the results of genome-wide association with peritoneal solute transfer, building on the study from Mehrotra et al., to the single-gene specific association of $A Q P 1$ with UF, could allow us to embark on the next steps of designing highly stratified clinical trials with the ultimate goal of optimizing treatment for individual patients on PD [1].

\section{Conclusion}

So, is my PET in my genes? The answer to that is an emphatic "partially." Clearly, peritoneal membrane characteristics are not predominantly determined by a few rare variants with large effect. But this is what characterizes "complex traits": they are defined by a combination of genetic and environmental factors. Even the genetically most advantageous "Ubermembrane" will not transport sufficiently, if scarred by infections. As discussed above, Mehrotra et al. calculate that roughly one fifth of the variability in PSTR is inherited, yet that this is driven by numerous variants, each with rather small effects [10]. In this regard, it is unfortunate that the study on UF only assessed variants in the candidate gene $A Q P 1$, rather than performing a GWAS. The advantage of genome-wide studies is that they are unbiased, free of assumptions about underlying mechanisms, and therefore have the power to discover the completely unexpected. The $p$-value for the association between rs2075574 in AQPI and UF was 0.007 , which is significant for a single association, but clearly not on a genome-wide level. Results of a GWAS are typically visualized in a "Manhattan plot," in which the $Y$-axis represents the negative decadic logarithm for the $p$-value. The smaller the $p$-value for a given association, the higher it plots on the $Y$-axis, and we consequently are looking for "skyscrapers," i.e., associations with a $p$-value $<5 \times 10^{-8}$. In contrast, a $p$-value of 0.007 would be lost in the mass of Brownstones and other low rises of a Manhattan plot. It is, of course, entirely possible that a GWAS of UF would have been similarly unsuccessful in identifying associations of genome-wide significance as it had been for PSTR. But even the suggestive associations in the PSTR GWAS have a $p$-value that is $2-3$ orders of magnitude smaller than that observed for rs 2075574 and UF. Thus, a GWAS of UF could have provided novel insights, perhaps even with regards to the molecular make-up of the small pores that mediate most of the peritoneal water transport.

At present, the results of these studies are emblematic for the state of using genetics for precision medicine of complex disorders: we still have a long way to go. Currently, we simply do not have the tools to implement precision medicine in PD treatment. But studies like these are small steps along that way, especially if followed up by a formal GWAS of UF, that could help identify more genetic variants of relevance. Stratifying clinical research cohorts by relevant genotypes may help us to make clinical trials in PD more effective, to move us along further towards personalized PD therapy.

\section{References}

1. Tsimberidou AM, Fountzilas E, Nikanjam M, Kurzrock R (2020) Review of precision cancer medicine: evolution of the treatment paradigm. Cancer Treat Rev 86:102019. https://doi.org/10.1016/j. ctrv.2020.102019

2. Bockenhauer D, Medlar AJ, Ashton E, Kleta R, Lench N (2012) Genetic testing in renal disease. Pediatr Nephrol 27:873-883. https://doi.org/10.1007/s00467-011-1865-2

3. Wuttke M, Kottgen A (2016) Insights into kidney diseases from genome-wide association studies. Nat Rev Nephrol 12:549-562. https://doi.org/10.1038/nrneph.2016.107

4. Rees L, Schaefer F, Schmitt CP, Shroff R, Warady BA (2017) Chronic dialysis in children and adolescents: challenges and outcomes. Lancet Child Adolesc Health 1:68-77. https://doi.org/10. 1016/S2352-4642(17)30018-4

5. Rippe B, Stelin G, Haraldsson B (1991) Computer simulations of peritoneal fluid transport in CAPD. Kidney Int 40:315-325. https://doi.org/10.1038/ki.1991.216

6. Devuyst O, Rippe B (2014) Water transport across the peritoneal membrane. Kidney Int 85:750-758. https://doi.org/10.1038/ki. 2013.250

7. Krediet RT, Lindholm B, Rippe B (2000) Pathophysiology of peritoneal membrane failure. Perit Dial Int 20:S22-S42

8. Rusthoven E, Krediet RT, Willems HL, Monnens LA, Schroder CH (2005) Sodium sieving in children. Perit Dial Int 25:S141-S142

9. Lambie M, Chess J, Donovan KL, Kim YL, Do JY, Lee HB, Noh H, Williams PF, Williams AJ, Davison S, Dorval M, Summers A, Williams JD, Bankart J, Davies SJ, Topley N; Global Fluid Study Investigators (2013) Independent effects of systemic and peritoneal inflammation on peritoneal dialysis survival. J Am Soc Nephrol 24:2071-2080. https://doi.org/10.1681/ASN.2013030314

10. Mehrotra R, Stanaway IB, Jarvik GP, Lambie M, Morelle J, Perl J, Himmelfarb J, Heimburger O, Johnson DW, Imam TH, Robinson B, Stenvinkel P, Devuyst O, Davies SJ; Bio-PD Consortium (2021) A genome-wide association study suggests correlations of common genetic variants with peritoneal solute transfer rates in patients with kidney failure receiving peritoneal dialysis. Kidney Int 100:1101-1111. https://doi.org/10.1016/j.kint.2021.05.037

11. Johnson RC, Nelson GW, Troyer JL, Lautenberger JA, Kessing BD, Winkler CA, O'Brien SJ (2010) Accounting for multiple 
comparisons in a genome-wide association study (GWAS). BMC Genomics 11:724. https://doi.org/10.1186/1471-2164-11-724

12. Sun H, Yang X, Liang L, Zhang M, Li Y, Chen J, Wang F, Yang T, Meng F, Lai X, Li C, He J, He M, Xu Q, Li Q, Lin L, Pei D, Zheng H (2020) Metabolic switch and epithelial-mesenchymal transition cooperate to regulate pluripotency. EMBO J 39:e102961. https:// doi.org/10.15252/embj.2019102961

13. Zhu P, Lv J, Yang Z, Guo L, Zhang L, Li M, Han W, Chen X, Zhuang H, Lu F (2014) Protocadherin 9 inhibits epithelial-mesenchymal transition and cell migration through activating GSK3 beta in hepatocellular carcinoma. Biochem Biophys Res Commun 452:567-574. https://doi.org/10.1016/j.bbrc.2014.08.101

14. Morelle J, Marechal C, Yu Z, Debaix H, Corre T, Lambie M, Verduijn M, Dekker F, Bovy P, Evenepoel P, Bammens B, Selgas R, Bajo MA, Coester AM, Sow A, Hautem N, Struijk DG, Krediet
RT, Balligand JL, Goffin E, Crott R, Ripoche P, Davies S, Devuyst O (2021) AQP1 promoter variant, water transport, and outcomes in peritoneal dialysis. N Engl J Med 385:1570-1580. https://doi. org/10.1056/NEJMoa2034279

15. Morelle J, Sow A, Fustin CA, Fillee C, Garcia-Lopez E, Lindholm B, Goffin E, Vandemaele F, Rippe B, Oberg CM, Devuyst $\mathrm{O}$ (2018) Mechanisms of crystalloid versus colloid osmosis across the peritoneal membrane. J Am Soc Nephrol 29:1875-1886. https://doi.org/10.1681/ASN.2017080828

Publisher's note Springer Nature remains neutral with regard to jurisdictional claims in published maps and institutional affiliations. 